\title{
Technological innovations for clinical sample storage in a Swedish Cervical Cytology Biobank: a review
}

This article was published in the following Dove Press journal:

Journal of Biorepository Science for Applied Medicine

2I August 2014

Number of times this article has been viewed

\author{
Nasrin Perskvist ${ }^{1,2}$ \\ 'Division of Laboratory Medicine, \\ Department of Pathology, Karolinska \\ Institute, ${ }^{2}$ Laboratory of Clinical \\ Pathology-Cytology, Karolinska \\ University Hospital, Huddinge, \\ Stockholm, Sweden
}

\begin{abstract}
The biological samples collected from women who have participated in cervical cancer screening constitute huge cohorts, providing the necessary statistical power to aid researchers in translating basic scientific discoveries into clinical applications. The availability of high-quality cytological specimens for diagnostic and research purposes requires the development of standardized methods for sample handling and long-term storage to enable the future use of specimens. The Swedish Cervical Cytology Biobank (SCCB) is an innovative solution for the biopreservation of gynecological cell samples. SCCB is the first national initiative for a prospective repository of liquid-based gynecological cell samples from women participating in organized and nonorganized cervical cancer screening. The successful nationalization of the SCCB is based on the three vital issues: 1) the system's flexibility to adapt to other regional systems; 2) system development, as based on a national collaboration between the university and county councils; and 3) stable government financing by the provider, BBMRI.se. The greatest advantage of a nationalized biobanking infrastructure is the tremendous potential to perform high-quality, multicenter research using the biobank's samples and the samples' national clinical records. We intend to share our experiences with biorepository communities to promote an understanding of and advances in opportunities to establish a disease-oriented and populationbased biobank that covers the health care needs of the entire nation.
\end{abstract}

Keywords: cervical cytology, automation solutions, national collaboration

\section{Introduction}

The Swedish National Board of Health and Welfare issues national guidelines for cervical cancer screening. According to these guidelines, it is recommended that all women undergo screening tests every 3 years from the age of 23-50 years, and every 5 years between the ages of 50-60 years. This testing collects 700,000 samples annually (http:// www.socialstyrelsen.se), and liquid-based cytology (LBC) is the recommended method for screening and human papilloma virus testing in Sweden. ${ }^{1}$ The biological samples collected from women who have participated in cervical cancer screening constitute huge cohorts, providing the necessary statistical power to aid researchers in translating basic scientific discoveries into clinical applications. Systematic biobanking of this cohort would greatly improve both clinical diagnostics and biomolecular research on cervical cancer and other diseases that affect women. The primary responsibility of biobanks is to collect biospecimens that truly reflect the local population, thereby promoting translational research that is applicable to the community. The availability of high-quality cytological specimens for diagnostic and research purposes requires the development of standardized methods for sample handling and long-term storage to enable the future use of specimens.$$
\text { sample handing and long-term storage to enable the future use of specimens. }
$$

\footnotetext{
Correspondence: Nasrin Perskvist Clinical Cytology Biobanking, Nationa Cervical Cytology Biobank, Karolinska Institute, Department of Pathology, F56, Karolinska University Hospital, Huddinge, S-I4I 86 Stockholm, Sweden Tel +46854288062

Fax +46854283702

Email nasrin.perskvit@ki.se
} 
The Swedish Cervical Cytology Biobank (SCCB; Karolinska University Hospital, Stockholm, Sweden) is an innovative solution for the biopreservation of gynecological cell samples. SCCB is the first national initiative for a prospective repository of liquid-based gynecological cell samples from women participating in organized and nonorganized cervical cancer screening. ${ }^{2}$

The development of the biorepository process, its application into a high-throughput robotic system, and the nationalization of the biobank has been government financed by the Swedish Research Council (http://www.vr.se) and regulated by the Biobanking and Molecular Resource Infrastructure of Sweden (BBMRI.se). BBMRI.se is part of the Karolinska Institute in Stockholm, Sweden.

\section{The preparatory phase}

\section{Initiation phase of the biobank}

The SCCB approach was initiated on April 2011 as a pilot project at Karolinska Hospital (Stockholm, Sweden) and relied on a collaboration with Karolinska University (Stockholm, Sweden). On August 2011, the Cervical Cytology Biobank, which was initiated as a pilot project by the Stockholm County Council, adopted a unified sample handling and storage process for the biopreservation of LBC cervical cell samples nationwide. ${ }^{2}$ Various county councils implemented the system across the country, and the SCCB was consequently created as a unified national biobanking system. To date, the biobank serves 12 county councils and processes more than $60 \%$ of the liquid-based gynecological cell samples obtained throughout Sweden. The SCCB headquarters are located at Karolinska University Hospital in Stockholm, Sweden.

\section{Legal aspects integrated with the biobank}

During the initiation phase, all of the legal aspects were taken into account and included in the design of the biobank system. These aspects included: 1) the "Biobank in Healthcare Regulation Act", which is intended to protect the integrity of the individual sample; 2) the "Health and Medical Services Act" on quality and safety standards in the handling of cell samples; 3) the "Patient Data Act" and the "Personal Data Act" for the protection of donors' privacy; and 4) the "Biobank Act", which regulates the use of the stored samples with the individual's consent within the health care system and research entities. Patients' informed consent was accordingly integrated into the design (http://www.socialstyrelsen. se/healthcare-visitors-sweden/about-swedish-healthcaresystem/healthcare-rules-regulations).

\section{The innovation of the biobanking process}

Our goal was to design a cell sample handling and storage strategy and to integrate the system with a highly automated process. A robotic process greatly increases the achievable throughput, reduces opportunities for operator error, and increases sample consistency and traceability. Prototype testing has been used to develop robust, affordable, and fully automated methods that prevent unexpected process bottlenecks or incompatibilities between technology and data. This biobanking procedure was advanced as an extension of current cytopathology practices in the laboratories. The sample processing procedures, defined in a series of standard operating procedures (SOPs), detail the largest amount of residual cervical cells to be collected for aliquoting and storage, the most advantageous final storage format, aliquoting volumes, storage processing, and storage temperature.

The cervical cells are collected into sample containers containing PreservCyt ${ }^{\circledR}$ (ThinPrep ${ }^{\circledR}$ Pap test; Hologic ${ }^{\circledR}$, Inc., Bedford, MA, USA), a methanol-based medium. The residual samples are then cycled from clinical laboratories to biobanks for processing after a diagnosis has been determined. The sample processing procedures were implemented into a robotic system in sequential steps, as follows: 1) scanning of 96-well storage plates $(0.5 \mathrm{~mL}$ Tracker $2 \mathrm{D}$ in Loborack-96w low cover, MPW52337BC3; Nordic Biolabs AB, Täby, Sweden) for the sample identification number; 2) aspiration of $4 \mathrm{~mL}$ of a cell suspension from sample containers into the conical tubes (Cat nr: 401201; Nordic Biolabs, AB) and sedimentation of cells in the bottom of the conical tubes; 3 ) aliquoting of $300 \mathrm{~mL}$ of sedimented cells as the final storage volume into the individual storage vials in the 96-well plate; and 4) labeling and manual transportation of the plate to the storage facility (Figure 1). The automated processing equipment handles individual sample containers, scans the sample labels, transmits the samples to the destination tubes, and assesses the liquid levels in both the intermediary conical tubes and final storage vials. This equipment increases the number of samples that can be processed in a given time and reduces the number of defects, providing quality assurance for the biobanking process design. Any defects are controlled either by halting the process or by issuing an alert to the operator.

The storage equipment is designed for operation at $-25^{\circ} \mathrm{C}$ to $-30^{\circ} \mathrm{C}$ and is connected to an automatic monitoring and alarm system that is readily available to the staff.

The design process, automation, and implementation of the biobank has been extensively discussed and described in our previous article. ${ }^{2}$ 


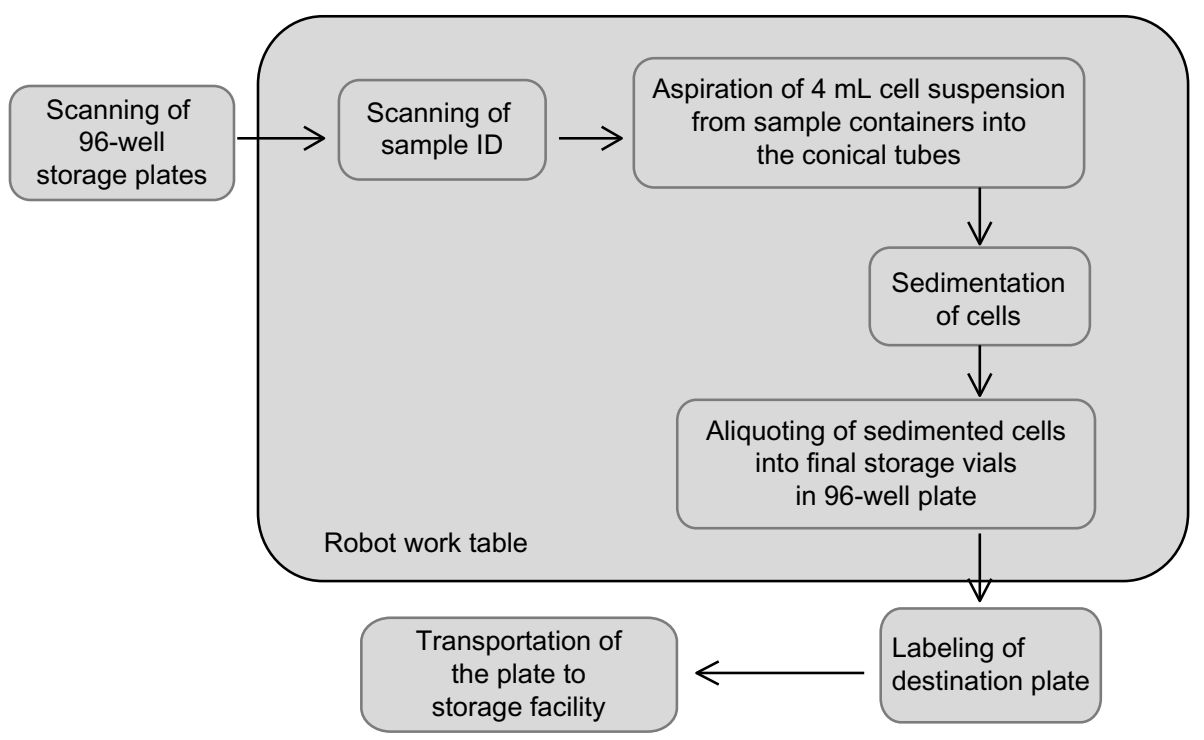

Figure I A summary of the automation of the sample handling process.

Notes: The sample processing procedures were implemented into a robotic system in sequential steps as follows: I) scanning of 96-well storage plates for the sample identification number; 2 ) aspiration of $4 \mathrm{~mL}$ of cell suspension from the sample containers into the conical tubes, and sedimentation of cells in the bottom of the conical tubes; 3 ) aliquoting of the sedimented cells at the final storage volume into the individual storage vials in the 96-well plate; and 4 ) labeling and manual transportation of the plate to the storage facility.

Abbreviation: ID, identification.

\section{LIMS integrated into the biobank}

The SCCB has implemented a robust, commercially available Laboratory Information Management System (LIMS) (LabWare-LIMS, http://www.labware.com) system that was initially developed for high-throughput biobank processing. Although this LIMS package is ideal for well-described and repeated operations given its logical data structure and compatibility with the robotic software database of the cytology biobank, it does require a special configuration so it can be aligned with the laboratory patient information system. The overall data structure and system has been designed and implemented alongside the biobank sample handling protocol according to several guiding principles.

The use of barcodes (one-dimensional on the patient sample containers and on the 96-well storage plate; twodimensional on the bottom of the storage vials) ensures the accuracy and traceability of all samples from the assessment center to the archive, and it links the samples to the patient information data systems at the correlated laboratories. Because the sample handling processes are fully automated, additional information - such as the time and date of processing and archiving, storage volume, and position in the biobank - can be attributed to each aliquot and linked to the individual donors. Participant confidentiality is of the utmost concern; all processed samples and their aliquots are exclusively identified by barcodes that can only be linked to the donors through a unique 12-digit two-dimensional bar code.

All authorized personnel are identified by LIMS through login information. Access to the entire database structure is restricted to the director of the biobank, who has overall responsibility. The backup storage is hosted on the Karolinska Institute server at the Karolinska Institute in Stockholm, Sweden.

\section{Biobank quality assurance and quality control}

The SCCB is an extension of LBC procedures used in clinical laboratories; therefore, a quality management system of a laboratory accreditation procedure (ISO 15189), including quality assurance and quality control programs, cover the full spectrum of biobanking operations. SOPs are documented according to the accreditation guidelines. The documented SOPs for the biobank describe how the tasks pertaining to repository operations should be handled by the staff members assigned to those specific responsibilities. These SOPs allow for uniformity and reproducibility in cervical cell sample handling.

SCCB quality control refers to the system that measures the quality of the processed samples in the biobank on a regular basis; the system also assesses instrument performance, quality, and calibration, and recommends equipment upgrades and replacement., ${ }^{2,3}$ 


\section{General costs of the biorepository of cell samples}

A cost-efficient infrastructure is a vital issue for the successful development and integration of the biobank into the health care system, as well as for providing samples to multiple researchers. During the preparatory phase of the biobank, a survey of the costs for construction and infrastructure components of the biobank was conducted, and an approximate cost of $€ 150,000$ was estimated (based on 2011 economics) in Sweden, excluding the costs of the biobank's operator, which differ regionally. The analysis indicated that the average cost of running LBC samples in the biobank is $€ 0.50 /$ sample based on the financial situation in 2011.

\section{Enhancement of biobank workflow}

The efficacy of the hospital-integrated biobank is mainly due to the increased ability of the biobank to provide samples to research groups without any risk of leaving insufficient sample volumes for donor care. Therefore, a complex intervention for the workflow enhancement of SCCB was performed at the headquarters 1 year after its inauguration. Briefly, the performance of the entire high-throughput system was validated by measuring the cell yield of the storage volume; the results were unsatisfactory after 1 year of sample collection and aliquoting. However, the results prompted a number of highquality technical interventions for workflow enhancement. Subsequently, the improved process was applied to the system and resulted in a significant increase in cell yield at the final volume. Increasing the storage volume from $300 \mu \mathrm{L}$ to $600 \mu \mathrm{L}$ served as a vital consequence of the intervention's processes. The enhancement procedures have been discussed in detail in our previously published article. ${ }^{3}$

\section{Nationalization process of the biobank infrastructure}

Health care in Sweden is organized regionally by counties, each of which has different high-quality and standardized methods for sample collection, transport, and diagnosis at clinical cytology laboratories (see http://www.vardguiden.se). Importantly, the issue of the nationalization of our biopreservatory process is built on the implementation of SOPs that are capable of adapting to different systems. ${ }^{4}$ Given the differences in the infrastructure and health care systems of various county councils in Sweden, these efforts were designed to develop a standardized framework of biobanking across the nation. Nationalization process of the biobank infrastructure has been extensively discussed and described in our recently accepted article. ${ }^{4}$ Briefly, the successful nationalization of the SCCB is based on the three vital issues: 1) system flexibility to adapt to other regional systems; 2) system development based on a national collaboration between the university and the county councils; and 3) stable government financing by the provider, BBMRI.se. The greatest advantage of the nationalized biobanking infrastructure has been the tremendous potential to perform high-quality, multicenter research using the biobank's samples and their related national clinical records. The SCCB network highlights the importance of mutual communication strategies for the exchange of knowledge in the field that benefits both the health care system and the researchers.

\section{Functioning of the biobank}

The goals of the SCCB are as follows: 1) create a national platform for the biobanking of gynecological cell samples despite regional differences; 2) ensure the performance of high-throughput sample handling for population-based cohorts; and 3) act as a hospital-integrated biobank that serves to provide patient care, as well as multiple research purposes with multiple researchers.

\section{Conclusion}

In conclusion, we intend to share our experiences with biorepository communities to promote an understanding of and advances in opportunities to establish a nationalized biobank that covers the health care needs of the entire nation.

\section{Acknowledgment}

The Swedish Cervical Cytology Biobank was developed and implemented with the support of BBMRI.se, which is funded by the Swedish Research Council.

\section{Disclosure}

The author reports no conflicts of interest in this work.

\section{References}

1. Fröberg M, Johansson B, Hjerpe A, Andersson S. Human papillomavirus 'reflex' testing as a screening method in cases of minor cytological abnormalities. Br J Cancer. 2008;99(4):563-568.

2. Perskvist N, Norman I, Eklund C, Litton JE, Dillner J. The Swedish cervical cytology biobank: sample handling and storage process. Biopreserv Biobank. 2013;11(1):19-24.

3. Perskvist N, Björklund C, Dillner J. A complex intervention for workflow enhancement at the Swedish cervical cytology biobank. Biopreserv Biobank. 2014;12(1):69-73.

4. Perskvist N. Norlin L, Dillner J. The Process of Moving from a Regionally Based Cervical Cytology Biobank to a National Infrastructure. Biopreserv Biobank. In press 2014. 


\section{Publish your work in this journal}

Journal of Biorepository Science for Applied Medicine is an international, peer-reviewed,

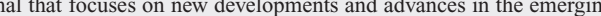
and evolving field of biorepository science. This includes biospecimen procurement processing, preservation, and banking for application to applied medicine. The Journal invites submission of manuscripts which address these aspects in addition to systems
logic, clinical throughput and ethical issues pertaining to application of biorepositories

and their effects on clinical medicine. The journal is characterized by the rapid reporting of reviews, original research, methodologies, technologies and analytics in this subject area. The manuscript management system is completely online and includes a very quick and fair peer-review system, which is all easy to use. Visit http://www.dovepress. com/testimonials.php to read real quotes from published authors.

Submit your manuscript here: http://www.dovepress.com/journal-of-biorepository-science-for-applied-medicine-journal 